\title{
Research Progress of the Influence of Acromegaly on Cardiovascular Diseases
}

\author{
Yalan Wang1*, Jiahe Xie ${ }^{2}$ \\ ${ }^{1}$ Gannan Medical University, Ganzhou 341000, Jiangxi Province, China \\ ${ }^{2}$ Department of Cardiology, The First Affiliated Hospital of Gannan Medical University, Ganzhou 341000, Jiangxi Province, \\ China \\ *Corresponding author: Yalan Wang, 2054499190@qq.com

\begin{abstract}
Acromegaly is a progressive disease caused by an increased in the levels of growth hormone (GH), followed by an increased in the levels of insulin-like growth factor-1 (IGF-1). Most cases are caused by GH-secreting pituitary adenomas. Long-term exposure to excessive GH would affect all systems of the body, of which the cardiovascular system has the highest incidence rate and mortality rate. This review discusses the influence of acromegaly on cardiovascular diseases and its treatment.
\end{abstract}

Keywords: Acromegaly; Cardiomyopathy; Hypertension; Arrhythmia; Vascular damage

Publication date: September 2021; Online publication: September 30, 2021

\section{Introduction}

Acromegaly is a rare disease. Its clinical features develop insidiously, and its diagnosis is often delayed ${ }^{[1]}$. The dysregulation of growth hormone secretion is usually caused by GH-secreting pituitary adenomas, thus leading to acromegaly - a disorder of disproportionate bone, tissue, and organ growth ${ }^{\text {[2] }}$. Due to the direct and indirect effects of GH and IGF-I on the cardiovascular system, involving a series of abnormalities of cardiac structure and function, acromegaly patients tend to develop cardiovascular complications, in which the mechanism is complex ${ }^{[3,4]}$. Due to the challenges in early diagnosis, it is often misdiagnosed clinically, resulting in an increased risk of mortality.

\section{Acromegalic cardiomyopathy}

In patients with acromegaly, long-term exposure to high levels of GH and IGF-1 will lead to acromegalic cardiomyopathy, which is characterized by concentric biventricular hypertrophy and diastolic dysfunction. In addition, progressive damage to the systolic function will lead to heart failure and arrhythmias, resulting in the deterioration of the cardiac function. The prolonged secretion of GH and IGF-1 in excess affects cardiac morphology and performance, resulting in concentric biventricular hypertrophy. This is due to the increase in the width of cardiomyocytes, indicating that new sarcomeres are closely juxtaposed. The most significant histological abnormalities are the increased in extracellular collagen deposition, myofibril disorder, monocyte necrosis, and lymphomonocyte infiltration; all of which will gradually damage the structure of the whole organ. This would eventually lead to systolic or diastolic dysfunction ${ }^{[5]}$. Excessive GH or IGF-1 causes an increase in sodium-water storage in the kidneys, sympathetic tension, insulin resistance, dyslipidemia, etc., resulting in the changes of cardiac structure and finally heart failure ${ }^{[6]}$. Several studies have shown that acromegalic cardiomyopathy may occur without the involvement of cardiovascular risk factors ${ }^{[7]}$. In fact, this type of cardiomyopathy seems to be specific for acromegaly. 
Some morphological changes can be reversed, and cardiac insufficiency can be improved by controlling the levels of GH.

Acromegalic cardiomyopathy has a complex pathophysiology. In the early stage, there is enhanced myocardial function, decreased peripheral vascular resistance, and increased cardiac output. In the midstage, it is characterized by myocardial hypertrophy, which leads to the proliferation of myocardial fiber tissue due to inflammatory reactions. This in turn results in progressive interstitial remodeling and deterioration of cardiac diastolic and systolic function. Advanced acromegalic cardiomyopathy is characterized by significant dilation of the chambers, severe systolic and diastolic dysfunction, as well as high peripheral vascular resistance, which eventually leads to congestive heart failure ${ }^{[8]}$. The exact mechanisms of short-term GH exposure and cardiac insufficiency as well as long-term GH exposure leading to final heart failure are unclear, although they seem to be associated with the development of myocardial fibrosis and inflammation. Generally, if the treatment is not successful for many years, concentric hypertrophy and diastolic dysfunction would develop, leading to diastolic heart failure, and the complications of diabetes and high blood pressure may further lead to the deterioration of the cardiomyopathy. The excessive cardiovascular risk associated with acromegaly has long been recognized [9]. It has been reported that transsphenoidal adenomectomy can reduce left ventricular mass index and improve diastolic function if the disease is controlled ${ }^{[10]}$. In patients treated with somatostatin analogues, the left ventricular mass index decreases rapidly; the impact on cardiac morphology and function as well as the diastolic filling can be appreciated after 6 months of treatment. After 6-12 months of treatment, the effect is very significant ${ }^{[11]}$, and the improvement of diastole and systole is more obvious not only in patients who have achieved disease control, but also in young patients with a short duration of GH and IGF1 hypersecretion before intervention ${ }^{[12]}$.

\section{Hypertension}

Hypertension is one of the most important negative prognostic factors affecting the mortality of patients with acromegaly. Although the exact prevalence is unclear, it is reported that $50 \%$ of these patients suffer from hypertension ${ }^{[13]}$. Excessive GH may lead to insulin resistance and smooth muscle cell hypertrophy, leading to an increased in vascular resistance. Excessive GH will also cause an increased in sodium-water reabsorption. Heavy plasma volume load and insulin resistance are related to renin-angiotensin-aldosterone system hyperactivity and impaired nitric oxide production, resulting in impaired vasodilation function; thus, the occurrence of hypertension through these mechanisms ${ }^{[14,15]}$. Experimental studies on acromegaly have shown that endogenous digitalis-like factors play a role in the regulation of body fluids and electrolytes. Therefore, high levels of growth hormone can trigger the continuous release of these factors and increase blood pressure ${ }^{[16]}$. There may be another contributing factor to hypertension in these patients. Most patients with acromegaly have obstructive sleep apnea syndrome, which leads to hypoxia. This increases the sympathetic tension; thus, the contraction of endothelial function eventually leads to hypertension ${ }^{[17]}$.

The relationship between the severity of hypertension and the levels of GH or IGF-1 has been investigated; however, the results have been inconsistent. A recent article analyzed this relationship, and its results showed that when the IGF-1 concentration is overtly high, the blood pressure level is positively correlated with the level of IGF-1, whereas if the IGF-1 level is within the normal range, the blood pressure level is inversely proportional to the IGF-1 concentration ${ }^{[18]}$. This analysis included several studies, most of which were performed in non-acromegaly patients and supported the direct relationship in a state of significantly elevated IGF-1. Acromegaly patients usually suffer from hypertension without any gender bias ${ }^{[19]}$. Due to the impact of hypertension on the incidence rate and mortality rate, early treatment of hypertension is essential. It has been shown that the reduction of GH level after successful treatment of acromegaly lowers blood pressure and significantly reduces the use of antihypertensives. 


\section{Arrhythmias}

Arrhythmias are relatively rare in patients with acromegaly. However, if they occur, they may be related to the changes in the structure of the heart, especially in cardiomyopathies ${ }^{[20]}$. ECGs and ambulatory ECGs have recorded ectopic beats, paroxysmal atrial fibrillation, paroxysmal supraventricular tachycardia, sick sinus syndrome, ventricular tachycardia, and even bundle branch blocks in patients with acromegaly, mainly during exercise ${ }^{[21]} .48 \%$ of patients with acromegaly have complex ventricular arrhythmias, while only $12 \%$ of non-acromegaly patients have this condition. The frequency of ventricular premature beats increases with the duration of acromegaly. The severity of ventricular arrhythmia is related to the quality of the left ventricle. It has been reported that the incidence of late potentials on ECG in patients with active arrhythmia is $56 \%$. This may induce arrhythmia and increase the QT interval variability in patients with acromegaly. In order to combat potentially life-threatening arrhythmias in acromegalic cardiomyopathy, implantable cardioverter defibrillator (ICD) has been used in patients as primary and secondary prevention of sudden cardiac death. The reason for the frequent occurrence of ventricular tachycardia among these patients is the presence of myocardial fibrosis and scars caused by acromegaly ${ }^{[22]}$.

\section{Vascular damage}

Studies have shown that the carotid intima-media thickness in patients with acromegaly is increased compared to healthy subjects ${ }^{[23]}$; however, there are several cases where healthy people also have an increased carotid intima-media thickness ${ }^{[24]}$. The incidence rate of coronary heart disease in patients with acromegaly varies widely in different countries. This may be related to the cardiovascular risk factors of atherosclerosis and coronary heart disease, such as age, gender, diet, etc. Cardiovascular risk factors have a greater impact compared to long-term exposure to GH or IGF-1 ${ }^{[25]}$. IGF-1 has a direct or indirect effect on immune cells, endothelial cells, and vascular smooth muscle cells. Endothelial nitric oxide synthase induces the production of endothelial nitric oxide, but in the absence of tetrahydrobiopterin or L-arginine, nitric oxide synthase is uncoupled to produce precursors of most reactive oxygen species rather than nitric oxide (NO). This would eventually lead to endothelial cell dysfunction, which is related to the occurrence and development of atherosclerosis ${ }^{[26]}$. By increasing the precursors of reactive oxygen species, reducing NO availability, and increasing the production of oxidized low-density lipoprotein, it promotes the formation of foam cells from mononuclear cells invading the vascular wall, which further aggravates the inflammation and damage ${ }^{[27]}$. The expression of nitric oxide and nitric oxide synthase decreases in patients with active acromegaly, and the expression of nitric oxide synthase and nitric oxide is negatively correlated with GH or IGF-1 levels ${ }^{[28]}$.

\section{Conclusion}

$\mathrm{GH}$ is mainly involved in regulating body growth, including cardiac development and function. It plays a direct or indirect role by stimulating its tissue effector, IGF-1. GH, IGF-1, and its binding peptides act directly or indirectly on the cardiovascular system through various secretory ways. Insufficient or excessive $\mathrm{GH}$ production would affect the incidence rate and mortality of cardiovascular diseases. Although drug therapy is beneficial to patients who do not need surgery, ultimately, the control of hormone levels would lead to a reduction in mortality, emphasizing the importance of early identification and treatment.

\section{Disclosure statement}

The authors declare that there is no conflict of interest. 


\section{References}

[1] Sisco J, van der Lely AJ, 2021, Towards an Earlier Diagnosis of Acromegaly and Gigantism. Journal of Clinical Medicine, 10(7).

[2] Melmed S, 2009, Acromegaly Pathogenesis and Treatment. The Journal of Clinical Investigation, 119(11): 3189-3202.

[3] Colao A, Ferone D, Marzullo P, et al., 2004, Systemic Complications of Acromegaly: Epidemiology, Pathogenesis, and Management. Endocr Rev, 25: 102-152.

[4] Colao A, Marzullo P, Di Somma C, et al., 2001, Growth Hormone and the Heart. Clin Endocrinol (Oxf), 54: 137-154.

[5] Arcopinto M, Bobbio E, Bossone E, et al., 2013, The GH/IGF-1 Axis in Chronic Heart Failure. Endocr Metab Immune Disord Drug Targets, 13(1): 76-91.

[6] Ramos-Leví AM, Marazuela M, 2019, Bringing Cardiovascular Comorbidities in Acromegaly to an Update. How Should We Diagnose and Manage Them?. Frontiers in Endocrinology, 10: 120.

[7] Mosca S, Paolillo S, Colao A, et al., 2013, Cardiovascular Involvement in Patients Affected by Acromegaly: An Appraisal. Int J Cardiol, 167(5): 1712-1718.

[8] Matta MP, Caron P, 2003, Acromegalic Cardiomyopathy: A Review of the Literature. Pituitary, 6(4): 203-207.

[9] Orme SM, McNally RJ, Cartwright RA, et al., 1998, Mortality and Cancer Incidence in Acromegaly: A Retrospective Cohort Study. United Kingdom Acromegaly Study Group. Journal of Clinical Endocrinology and Metabolism, 83: 2730-2734.

[10] Minniti G, Moroni C, Jaffrain-Rea ML, et al., 2001, Marked Improvement in Cardiovascular Function After Successful Transsphenoidal Surgery in Acromegalic Patients. Clin Endocrinol (Oxf), 55: 307-313.

[11] Baldelli R, Ferretti E, Jaffrain-Rea ML, et al., 1999, Cardiac Effects of Slow-Release Lanreotide: A Slow-Release Somatostatin Analog in Acromegalic Patients. J Clin Endocrinol Metab, 84: 527-532.

[12] Colao A, Marzullo P, Cuocolo A, et al., 2003, Reversal of Acromegalic Cardiomyopathy in Young but Not in Middle-Aged Patients After 12 Months of Treatment with the Depot Long-Acting Somatostatin Analogue Octreotide. Clin Endocrinol (Oxf), 58: 169-176.

[13] Isgaard J, Arcopinto M, Karason K, et al., 2015, GH and the Cardiovascular System: An Update on a Topic at Heart. Endocrine, 48(1): 25-35.

[14] González B, Vargas G, de Los Monteros ALE, et al., 2018, Persistence of Diabetes and Hypertension After Multimodal Treatment of Acromegaly. Clin Endocrinol Metab, 103(6): 2369-2375.

[15] Mosca S, Paolillo S, Colao A, et al., 2013, Cardiovascular Involvement in Patients Affected by Acromegaly: An Appraisal. Int J Cardiol, 167(5): 1712-1718.

[16] Deray G, Rieu M, Devynck MA, et al., 1987, Evidence of an Endogenous Digitalis-Like Factor in the Plasma of Patients with Acromegaly. N Engl J Med, 316(10): 575-580.

[17] Attal P, Chanson P, 2010, Endocrine Aspects of Obstructive Sleep Apnea. Clin Endocrinol Metab, 95(2): 483-495.

[18] Schutte AE, Volpe M, Tocci G, et al., 2014, Revisiting the Relationship between Blood Pressure and Insulin-Like Growth Factor-1. Hypertension, 63: 1070-1077.

[19] Vitale G, Pivonello R, Auriemma RS, et al., 2005, Hypertension in Acromegaly and in the Normal Population: Prevalence and Determinants. Clin Endocrinol (Oxf), 63: 470-476.

[20] Auriemma RS, Pivonello R, De Martino MC, et al., 2013, Treatment with GH Receptor Antagonist in Acromegaly: Effect on Cardiac Arrhythmias. Eur J Endocrinol, 168(1): 15-22. 
[21] Rodrigues EA, Caruana MP, Lahiri A, et al., 1989, Subclinical Cardiac Dysfunction in Acromegaly: Evidence for a Specific Disease of Heart Muscle. Br Heart J, 62: 185-194.

[22] Bardy GH, Smith WM, Hood MA, et al., 2010, An Entirely Subcutaneous Implantable CardioverterDefibrillator. N Engl J Med, 363: 36-44.

[23] Oflaz B, Pamukçu M, Meriç F, et al., 2010, Investigation of Early Atherosclerotic Changes in Acromegalic Patients. International Journal of Clinical Practice, 64(1): 39-44.

[24] Paisley AN, Banerjee M, Rezai M, et al., 2011, Changes in Arterial Stiffness but Not Carotid Intimal Thickness in Acromegaly. The Journal of Clinical Endocrinology \& Metabolism, 96(5): 1486-1492.

[25] Anagnostis P, Efstathiadou Z, Gougoura S, et al., 2013, Oxidative Stress and Reduced Antioxidative Status, along with Endothelial Dysfunction in Acromegaly. Horm Metab Res, 45(4): 314-318.

[26] Hong F, Liang X, Liu W, et al., 2019, Roles of eNOS in Atherosclerosis Treatment[J]. Inflammation Research, 68(6): 429-441.

[27] Boero L, Cuniberti L, Magnani N, et al., 2010, Increased Oxidized Low Density Lipoprotein Associated with High Ceruloplasmin Activity in Patients with Active Acromegaly. Clinical Endocrinology, 72(5): 654-660.

[28] Ronconi V, Giacchetti G, Mariniello B, et al., 2005, Reduced Nitric Oxide Levels in Acromegaly: Cardiovascular Implications. Blood Pressure, 14(4): 227-232. 